\title{
Interférences
}

Ars scribendi

$11 \mid 2018$

Poésie latine et miroir du prince

\section{L'âge d'or sous le règne des Savoia : miroir du Prince, miroir de l'Italie dans Ad Victorem regem de Giovanni Pascoli}

Sarah Orsini

\section{(2) OpenEdition}

Journals

Édition électronique

URL : http://journals.openedition.org/interferences/6572

DOI : 10.4000/interferences.6572

ISSN : $1777-5485$

Éditeur

HiSoMA - Histoire et sources des Mondes antiques

Référence électronique

Sarah Orsini, «L'âge d'or sous le règne des Savoia : miroir du Prince, miroir de l'Italie dans Ad Victorem regem de Giovanni Pascoli », Interférences [En ligne], 11 | 2018, mis en ligne le 26 juillet 2018, consulté le 15 septembre 2020. URL : http://journals.openedition.org/interferences/6572 ; DOI : https://doi.org/ 10.4000/interferences.6572

Ce document a été généré automatiquement le 15 septembre 2020.

Tous droits réservés 


\title{
L'âge d'or sous le règne des Savoia : miroir du Prince, miroir de l'Italie dans Ad Victorem regem de Giovanni Pascoli
}

\author{
Sarah Orsini
}

Giovanni Pascoli, Ad Victorem Regem - anno Italiae liberatae L

Hic tu manebis, conditor, optime.

Terramque frustra magnus equus fodit,

tamquam peracturus diremptam

nocte uiam dubiamue pugnam.

Hic tu manebis, conditor, optime.

Terramque frustra magnus equus fodit,

tamquam peracturus diremptam

nocte uiam dubiamue pugnam.

Consistat. Absunt iam litui procul.

Vanescit omnis clamor. Obit furor

ceu sidus infestum. Renidet

Pax roseo ueneranda caelo.

Consistat. Absunt iam litui procul.

Vanescit omnis clamor. Obit furor

ceu sidus infestum. Renidet

Pax roseo ueneranda caelo.

Haec ara Pacis, fortis eques, tuae.

Te rura caelum nutriat augure

frugesque sol felix ab aruis

eliciat latiusque raster.

Ac temptet altis oppida sibilis

foedetque fumis ignipotens labor

dum dulcis umbra lar et unum

quemque suus iuuet hortus aura.

Maestos sequatur carior exsules

antiqua mater nec sinat immemor 
errare per diuersa terrae

soliuagos inopesque iuris.

Nostri reuisant litora nauitae,

quodcumque nobis paruit aut patet

per nos, et acres classe certent

ueliuolum perarare caelum.

Magnis iuuentus maior auis suo

fungatur ultro munere nec bonas

detrectet artes nec pudorem et

casta modum ueteresque mores.

Aedes nitenti congreget atrio

paruos Quirites et docili beet

uirtute. Quos auscultet altrix

Italia Italiam canentes.

Hic tu manebis, pacifer, aereus.

Frustraque terram magnus equus cauet.

Quod si Latini, Victor, olim

« Mars uigila! clupeos mouentes

dicemus, armis cum fragor ingruat

circum recussis, clangor ad ultimum

certamen erumpentis ensis

quo placidae tremat ara Pacis,

clangore longo tunc iterabitur

cliuoque sacro Ianiculum procul

atque ensis ensi duxque regi,

quod solitus, resonabit: «Adsum!»

et fluctuantem subter equis suam

prorsus uidebunt Italiam mari

terraque supremae perennesque

excubiae geminusque uindex.

« Pour le Roi Victor (au cinquantenaire de la libération de l'Italie) »

«Ici tu resteras, fondateur, parfaitement.

Et la terre, en vain le grand cheval la retourne, comme s'il devait aller au bout de la route

discontinue, de nuit, ou achever le combat incertain.

Qu'il reste immobile. Il n'y a plus de trompettes au loin.

S'évanouit tout cri. Disparaît la fureur

comme une étoile hostile. Rayonne

la Paix vénérable dans le ciel rose.

Cet autel de la Paix, vaillant chevalier, est tien.

Que sous tes augures le ciel nourrisse les campagnes,

et que les moissons, le soleil fécond, des champs

les fasse sortir, ainsi que le râteau latin.

Et qu'il atteigne les villages avec ses sifflets aigus,

et les souille de sa fumée, maître du feu, le travail, pour que de la douce ombre de sa maison, chacun profite, ainsi que de la richesse de son propre jardin.

Les tristes exilés, qu'elle les suive, si chérie,

l'antique mère, et qu'elle ne permette pas, les oubliant, qu'ils errent aux quatre coins du monde solitaires et privés de droits.

Que nos marins reviennent voir nos rivages, quel que soit celui qui s'ouvre à nous ou s'étend devant nous, et que fougueux avec leur flotte ils luttent pour sillonner le ciel qui se traverse à la voile. 
Que la jeunesse, plus grande que les grands oiseaux,

s'acquitte spontanément de sa tâche et les beaux-

arts qu'elle ne les rejette pas, ni la pudeur ni,

chaste, la mesure ainsi que les coutumes anciennes.

Qu'une maison rassemble dans une lumineuse salle d'entrée

les petits Quirites et les dote d'une docile

vertu. Et qu'elle les écoute, la nourrice

Italie, c'est l'Italie qu'ils chantent.

Ici tu resteras, pacificateur, homme d'airain

Et en vain, la terre, le grand cheval la creuse.

Or si nous les Latins, Victor, un jour

nous disons "Mars, debout!" en agitant

nos boucliers, quand le fracas des armes entrechoquées

envahira les environs, le sifflement de l'épée

qui pour la dernière lutte s'élance,

pour faire trembler l'autel de la tranquille Paix,

par un long sifflement alors sera repris,

et à la colline sacrée, le Janicule au loin

et l'épée à l'épée, et le général au roi,

comme d'habitude, répondra haut et fort "Présent!"

et agitée sous la houle des chevaux, leur

Italie, ils la verront pleinement, eux qui sur la mer

et la terre, sont de suprêmes et pérennes

sentinelles, les deux défenseurs. »

[trad. personnelle]

1 Le texte que nous allons aborder est une ode de Giovanni Pascoli intitulée Ad Victorem regem - anno Italiae liberatae $L^{1}$. Constituée de strophes alcaïques ${ }^{2}$, elle fut publiée le 5 juin 1911 dans le quotidien bolognais Il Resto del Carlino, à l'occasion de l'inauguration de l'Autel de la Patrie, autrement connu sous le nom de Vittoriano, un monument national comportant, au centre de son entrée, la statue équestre de Victor Emmanuel II, premier roi de l'Italie unifiée. L'édifice, commandé en 1885 par son successeur Humbert $\mathrm{I}^{\mathrm{er}}$, est l'emblème du Risorgimento, du royaume d'Italie et de la nation italienne. Les travaux ont été achevés sous Victor Emmanuel III. C'est donc lui qui inaugura l'Autel de la Patrie et sa statue le 4 juin 1911, à la suite de la célébration du cinquantenaire de l'Italie unifiée, c'est-à-dire 50 ans après que Victor Emmanuel II a été proclamé Roi d'Italie et 40 ans après l'annexion de Rome au Royaume d'Italie.

Lors de sa première publication, l'ode était intitulée Victori regi ${ }^{3}$, elle fut ensuite publiée par Zanichelli dans les mois qui suivirent sous son titre actuel ${ }^{4}$, et à titre posthume en 1917 par Ermenegildo Pistelli au sein d'une section qu'il appela Lyra romana et qu'un second éditeur, Adolfo Gandiglio, renomma Poematia et Epigrammata ${ }^{5}$ en 1930. Considérée comme un poème mineur, elle n'a, comme nombre des textes de cette section, jamais été étudiée en détail.

3 Ce poème s'inscrit pourtant dans une période très intense pour Pascoli en matière de composition, une époque que Maria Pascoli, sa sœur et biographe, appelle "l'année sacrée$^{6}{ }^{\prime}$, celle de la préparation de la publication des Poemi del Risorgimento ${ }^{7}$, de la publication des deux Hymni bilingues in Romam et in Taurinos ${ }^{8}$ et du discours La grande proletaria si è mossa9 $a^{9}$, prononcé à Barga en novembre 1911 en faveur de l'intervention militaire en Libye. Ainsi, la maison Zanichelli, qui soutenait Pascoli dans l'édition de ses recueils de poésie italienne, publie en 1911 trois textes en latin, ayant le point commun de célébrer l'histoire de l'Italie. Cette année 1911 peut être considérée comme l'aboutissement d'un cycle de poésie "épico-historique ${ }^{10}$ » commencé en 1906 avec les 
recueils italiens Odi e Inni, Le canzoni di Re Enzio et Poemi Italici ${ }^{11}$, et le poème Post Occasum Urbis que Pascoli a retravaillé pour écrire l'Hymnus in Romam dont le style lyrique, riche d'exaltation patriotique, caractérisa la poésie de la fin de sa vie. Pascoli a donc assumé à cette époque le rôle de poeta vate, successeur de Carducci pour célébrer la patrie ${ }^{12}$, et d'homologue de Victor Hugo, avec lequel il correspondait. Pascoli dit d'ailleurs à propos de cette période :

Comincia ora la mia vita di poeta e di scrittore e di educatore ${ }^{13}$.

Or, c'est précisément parce qu'il mêle les fonctions de célébration et d'éducation que nous avons choisi de nous intéresser au poème Ad Victorem regem dans le cadre de cette journée d'étude sur le miroir du Prince. Ce texte semble en effet avoir une forme adaptée pour être étudié comme tel, à un détail près : le Prince auquel il s'adresse à la deuxième personne est mort depuis vingt-trois ans.

Pour qui écrire un tel texte si ce n'est pas pour le dirigeant? Dans quelle mesure ce texte peut-il être considéré comme un miroir du Prince? Quels projets et quelles valeurs doivent porter le gouvernement et la nation italienne selon ce texte? Nous nous attacherons à montrer dans cet article que Pascoli extrait les codes du miroir du Prince à la fois pour immortaliser la figure du père fondateur en un monument de l'histoire italienne et pour véhiculer auprès du public ses souhaits idéologiques concernant l'avenir de la jeune nation italienne, en s'inspirant notamment des topoi établis par les poètes augustéens.

\section{L'épineuse question du destinataire}

\section{Un texte adressé au Prince, oui ... mais lequel ?}

Comme son titre l'indique, sous ses deux formes, le poème est dédicacé «Au roi Victor ». Or il y a deux Victor dans l'histoire de l'Italie, et la date anniversaire de l'unité italienne entretient la superposition des deux destinataires, réunis en une même figure de "Père de l'Italie ». Certes, le sujet de la première strophe, tu manebis, conditor, est Victor Emmanuel II. Mais ce dernier est décédé en 1897, et le poème s'adresse à une statue de bronze (aereus, v. 33). Il ne peut donc être destinataire du texte que sous forme d'hommage et de symbole. Ce n'est donc pas à lui que le texte va proposer un enseignement sur l'art de gouverner.

En ce qui concerne Victor Emmanuel III, nous n'avons trouvé aucune trace de correspondance avec Pascoli dans l'Archive de Castelvecchio, si ce n'est le brouillon d'une lettre de 1905 demandant grâce pour Linda Murri, condamnée pour complicité dans le meurtre de son mari, une lettre qui n'a jamais été envoyée. Cependant, Pascoli est une personnalité publique, fréquemment sollicitée pour des discours ou des expertises sur l'enseignement. Il a déjà dédicacé en 1898 un poème à Marguerite de Savoie, épouse d'Humbert I ${ }^{\mathrm{er}}$, mère de Victor Emmanuel III et protectrice des arts : Margarethae Sabaudae Italorum Reginae, traduction en latin d'un poème d'Alfredo Baccelli, «Sul Monte Rosa », au sujet d'un observatoire portant le nom de la reine et visité par celle-ci en 1898. Il est également l'auteur d'épigraphes gravées à l'occasion des visites de la reine à Pienza (1904) - elle est alors appelée "prima Regina d'Italia unita e indipendente » - et à Domodossola (1906), pour l'inauguration d'un "ospizio degli emigranti ». Cependant, l'épigraphe qui nous intéresse tout particulièrement est celle de 1911 en l'honneur de la visite de Victor Emmanuel III et de son épouse Hélène à 
la manufacture des tabacs de Lucques à l'automne 1910, qui présente des thématiques communes avec notre poème : la paix après la guerre, la justice sociale, le travail, le peuple italien :

Non più l'azza e lo stocco dalle turrite castella alpine / sì da Roma reco l'ulivo e la rosa / venni a voi nell'anno quinquagesimo dell'anno dei mille/ lavoratori, lavoratrici / nelle persone / del Re giusto nepote del Re liberatore / l'uno Vittorio e l'altro / e della sua compagna Regina / figlia di principi sacerdoti e guerrieri / madre di buoni e belli italici figli / Chiesi con la voce soave di Lei - i vostri figli? / risposi con la voce di Lui ferma e grave / I figli vedranno ciò che i padri - non videro ancora / dai duri sassi cresciuto l'olivo, dagl'irti pruni fiorita la rosa / dalle armi la pace, dal lavoro la felicità ${ }^{14}$.

«Ce ne sont plus la hache ni l'épée des châteaux alpins munis de tours / oui, de Rome j'apporte l'olivier et la rose / je vins à vous lors du cinquantenaire de l'année des mille / travailleurs, travailleuses / dans les personnes du Roi juste, petit-fils du Roi libérateur / l'un Victor tout comme l'autre / et de sa compagne la Reine / fille de princes prêtres et guerriers / mère de bons et beaux enfants italiques / Je demandai avec la voix suave de la Reine "vos enfants?" / je répondis avec la voix du Roi ferme et grave / "Ces enfants verront ce que leurs pères n'ont pas pu encore voir / une fois que des durs cailloux aura poussé l'olivier, que des pruniers hirsutes aura fleuri la rose / des armes la paix, du travail le bonheur" » [trad. personnelle].

Cette épigraphe est postérieure à la visite du roi, qui a certes pu en avoir connaissance. De même, le poème Ad Victorem regem fut publié dans un journal bolognais, ce qui nous incite à penser que Victor Emmanuel III ne l'a pas lu, du moins pas immédiatement, et qu'il était destiné à un public local. Le roi Victor serait donc un destinataire symbolique, qu'il s'agisse du grand-père ou du petit-fils.

\section{Un lectorat instruit}

Les miroirs du Prince ont cependant un public qui dépasse le seul individu à la tête du pouvoir. En effet, si la transmission manuscrite nous a permis d'accéder à de tels textes, c'est qu'ils étaient diffusés au-delà des seules familles régnantes. Les poèmes de célébration, ancêtres des miroirs du Prince, à partir de l'époque augustéenne, étaient prononcés publiquement et appréciés par les élites ${ }^{15}$. Assurément, le lectorat du poème de Pascoli connaît le latin, ce qui nous laisse entendre qu'il est issu de l'élite culturelle.

9 La première publication est certainement accueillie par le milieu intellectuel de Bologne, lecteur potentiel du Resto del Carlino, notamment par les collègues et étudiants de Pascoli. Le texte remplit alors la fonction d'un poème de circonstance, attestant la volonté de soutenir, même de loin, un événement majeur de l'histoire italienne, en en accompagnant le compte rendu du journal.

Quant à la publication chez Zanichelli, elle vise un public plus large, national mais aussi européen, qui connaît la poésie latine de Pascoli par le concours annuel de poésie latine d'Amsterdam. C'est à un public de ce genre que pense Pascoli dans son discours Un poeta di lingua morta ${ }^{16}$ (1898). Dans ce texte, il justifie le choix d'écrire en latin à la fin du $\mathrm{XIX}^{\mathrm{e}}$ siècle à travers un hommage au poète latiniste Diego Vitrioli :

devo credere di esser per avere più intenditori, in tutto il mondo, del mio latino,

che nella sola Italia, del mio italiano.

De nombreux ambassadeurs de pays alliés étant présents lors de l'inauguration de l'Autel de la Patrie, cet événement n'était certainement pas anodin pour les pays voisins et devait être suivi de près dans la presse internationale. Le poème de Pascoli pouvait constituer une occasion de célébrer la grandeur de l'Italie et de réaffirmer 
celle-ci dans les rapports internationaux. En outre, Pascoli correspond avec des membres de la classe dirigeante et leur adresse fréquemment ses poèmes latins (on compte parmi eux F. Martini, ancien ministre de l'Instruction publique, et son secrétaire G. Biagi, F. Barnabei, député et membre du Conseil d'État, ainsi que des préfets et maires de localités dans lesquelles il a prononcé des discours ou enseigné). Ce texte n'a toutefois pas été prononcé publiquement lors de l'inauguration devant les notabilités, il est donc d'abord destiné à être lu par la société civile. Nous précisons aussi qu'il s'agit d'une élite plus intellectuelle que financière, Pascoli étant l'incarnation même de l'enseignant de classe moyenne qui a été nommé dans diverses régions d'Italie et a accédé avec difficulté à un poste stable et à la propriété. Pascoli est d'ailleurs rattaché au jeune ministère de l'Instruction publique, qui développe sur le territoire italien des dynamiques d'alphabétisation globale mais aussi d'ascension sociale depuis la loi Casati (1859). Pascoli ayant écrit deux anthologies latines pour l'école, Lyra et $E_{p o s}{ }^{17}$, il ne serait pas étonnant qu'il espère être lu de la jeunesse issue de l'école italienne (ou des pays voisins qui connaissent la même dynamique).

11 Enfin, ce texte s'inscrit dans un réseau d'autres poèmes latins que Pascoli avait pour projet de reprendre et de publier dans un recueil accompagné d'une traduction séparée, une silloge completa de ses poèmes classés pour retracer l'histoire de Rome et du peuple romain des origines à la Rome contemporaine, un projet probablement inspiré de la Légende des siècles de Victor Hugo. Malade en 1911, il n'a pas mené son projet à terme, mais il a publié les deux Hymnes à Rome et à Turin, en latin et en italien, retraçant l'histoire de ces villes jusqu'à l'unité italienne. Ainsi, nous pouvons supposer que Pascoli souhaitait soit traduire Ad Victorem Regem en italien pour en étendre le public national, soit traduire des poèmes qui lui font écho, à savoir Post occasum urbis et l'Hymnus in Romam, ou encore Moretum ${ }^{18}$ (qui célèbre la petite propriété et la morale rustique) et $E c \log a \mathrm{XI}^{19}$ (qui met en scène Virgile rêvant de justice sociale). Cela témoigne de son désir de partager avec le public italien son point de vue de poeta vate sur la société et la nation italiennes à travers les âges, rédigé dans ce qui représente dans la poésie pascolienne les deux versants d'une même langue: le latin pour reconstituer les âges anciens, l'italien pour décrire le milieu contemporain, «fonte e foce dello stesso fiume ${ }^{20} »$.

12 Ainsi ce poème, publié sur deux supports bien différents, un journal local et une édition nationale à portée européenne, a rencontré un lectorat restreint par la nécessité de comprendre le latin mais varié en termes de répartition géographique et sociale. Puisque Pascoli ne s'adresse à Victor Emmanuel que de façon symbolique, il ne cherche pas à l'influencer directement. Par ce poème de célébration, il transmet donc ses idéaux concernant l'État à ses lecteurs tout en construisant son texte comme un miroir du Prince, en héritier des poètes augustéens.

\section{Un poème de célébration qui reprend les codes littéraires du miroir du Prince}

\section{Célébrer et exhorter : un éloge en vers d'inspiration horatienne}

Ce texte est une ode dédicatoire de circonstance et, en cela, Pascoli s'inspire de son modèle favori, Horace. Nombreuses sont en effet les odes que ce dernier adresse à Auguste (Hor., carm. 1, 2; 1, 12; 4, 5; 4, 14 et 4, 15). Pascoli en reprend la double 
dynamique d'exhortation et de célébration caractéristique des éloges en vers que nous pouvons observer notamment dans carm. 4, 5 et dans le Chant séculaire ${ }^{21}$. En effet, le roi représenté par la statue équestre est appelé par des termes élogieux (conditor, pacifer, fortis eques), comme pour célébrer son triomphe, mais il reçoit également des prières puisque les subjonctifs d'ordre (nutriat, eliciat, temptet, foedet) sont placés sous ses augures (te augure).

14 En publiant ce poème à une date anniversaire et pendant un événement public, Pascoli s'inscrit encore dans la continuité d'Horace ainsi que des panégyristes impériaux comme Claudien, dont les panégyriques sont prononcés à l'occasion de l'accès au consulat du dirigeant qu'il loue. Cet éloge est donc également, dans un contexte de commémoration, l'occasion de dresser le bilan des réalisations du roi d'Italie, de la nation italienne et de ce qu'il reste à accomplir.

15 Ce texte reprend ainsi les codes de la poésie d'éloge adressée au dirigeant. Or, bien que la notion de miroir du Prince s'applique de façon stricte surtout à des textes en prose postérieurs au XII ${ }^{\mathrm{e}}$ siècle, cette poésie peut être considérée comme un ancêtre du miroir du Prince ${ }^{22}$ puisqu'elle construit une figure idéale du dirigeant, un reflet exemplaire à tendre à son destinataire.

\section{Construire une figure du Prince}

Cependant, ce n'est pas au roi que le poète s'adresse, mais à sa statue, c'est-à-dire à un symbole. En décrivant la statue équestre, Pascoli propose sa représentation de la figure du Prince, qui se superpose à celle donnée par la statue.

\section{La statue équestre de Victor Emmanuel II : symbole du Risorgimento et emblème de l'Italie}

17 D'après Diane Bodart, la statuaire, comme le portrait, est à « double titre comparable à un miroir du prince» parce qu'elle est censée «réfléchir la figure naturelle du souverain avec la même exactitude que l'instrument optique, mais aussi révéler en sa personne l'idée archétypale du prince ${ }^{23} »$. Ce qu'elle écrit à propos de la Renaissance semble vrai aussi dans l'Antiquité et à l'époque de Pascoli, puisque Victor Emmanuel II est représenté en habit militaire, son épée contre le flanc du cheval, immortalisé dans son retour triomphal du combat. Nous pensons que cette statue équestre est inspirée de celle de Marc Aurèle au centre de la place du Capitole ${ }^{24}$, à quelques pas de l'Autel de la Patrie, notamment en ce qui concerne la posture du cheval, au pas avec la patte levée. Cependant, Marc Aurèle est représenté en civil, sans arme, la main levée en signe de paix, un geste probablement inspiré de d'œuvres plus anciennes, puisqu'on trouve une statue équestre incomplète d'Auguste dans la même posture au musée national d'Archéologie d'Athènes. Ainsi, même si la statue de Victor Emmanuel II reprend les codes des statues équestres antiques, elle ne véhicule pas la même image du Prince: tandis qu'Auguste et ses successeurs sont représentés en garants de la paix, la grande réalisation du roi, ce sont ses conquêtes d'unification.

La description de la statue équestre par Pascoli s'inscrit aussi dans la continuité des modèles anciens. Tout d'abord, en utilisant le procédé de l'ekphrasis, caractéristique des épopées antiques, Pascoli dresse un portrait épique en mouvement du premier roi d'Italie. Celui-ci est en effet représenté comme s'il était vivant, et les titres qui le 
qualifient résument ses réalisations : Pascoli le nomme conditor pour sa fondation du royaume d'Italie, fortis eques pour les guerres d'indépendance qu'il a menées, et pacifer afin d'en faire le garant de la paix après la guerre. Ces titres le placent donc dans la continuité des fondateurs Énée et Romulus mais aussi d'Auguste. Quant au cheval, il est figé dans le mouvement: il reste en place (terramque frustra magnus equus fodit; consistat) tout en semblant s'élancer (tamquam peracturus diremptam / nocte uiam dubiamue pugnam), comme la statue réelle et celle de Marc Aurèle. Or, c'est le commentaire sur la posture du cheval qui évoque les conquêtes militaires dirigées par Victor Emmanuel II. En effet, en plus des combats explicitement mentionnés, les routes symbolisent l'expansion militaire dans la poésie patriotique de Pascoli, et c'est particulièrement clair dans l'Hymnus in Romam, composé au même moment, dans lequel celles-ci s'élancent à travers le monde (v. 131 sq.) puis servent de chemin aux légions (v. 143 sq.). Le cavalier quant à lui est associé dans l'Hymnus aux guides qui dirigeaient les Romains dans leurs expéditions, incarnés par Romulus et Rémus que Pascoli représente réconciliés dans la mort ${ }^{25}$ et qui se confondent avec Castor et Pollux ${ }^{26}$ pour conduire les voyageurs et les troupes (Hymnus in Romam, v. 159-166) :

At divi pugnis aderant in nube gemelli

quos oculis vidit nemo, tantumque licebat

stridentes inter lituos raucasque tubas et

clamorem fremitumque feros audire sub auras

hinnitus. Alter tamquam sol aureus, alter

noctis erat similis maesta circumdatus umbra.

Sublimes in equis simul immortalibus ibant

florentes iuvenes aeratas ante cohortes.

"Mais, divins, assistaient au combat, dans un nuage, des jumeaux

que de ses yeux personne ne vit, et il était seulement possible

entre les cors stridents, les rauques trompettes et

les cris et le grondement, d'entendre, sauvages, sous les vents

des hennissements. L'un était comme le soleil doré, l'autre,

à la nuit semblable, était entouré d'une triste ombre.

Sublimes sur leurs chevaux immortels, ensemble ils avançaient

ces jeunes gens en fleur, à l'avant des cohortes revêtues d'airain. »

[trad. personnelle]

On retrouve cette image des deux guides à la fin de Ad Victorem regem. Pascoli y mentionne la statue de Garibaldi sur le Janicule (les photos des deux statues sont d'ailleurs placées en haut des deux pages du poème dans l'édition Zanichelli) et appelle le général et le roi supremae perennesque/ excubiae, "suprêmes et pérennes sentinelles ", et geminus uindex, les associant ainsi à Romulus et Rémus et aux Dioscures.

Cependant cette description est très brève (environ huit vers répartis sur l'ensemble du poème), et en s'adressant au roi à la deuxième personne, Pascoli mêle à la description les codes du discours funèbre par la représentation de l'immobilité de la statue et la répétition de l'expression Hic tu manebis, qui ouvre et ferme le poème aux strophes 1 et 9. Cette expression au futur inscrit la figure du roi aereus dans l'immortalité. L'emploi de ce temps dans les deux dernières strophes du poème fait des deux héros du Risorgimento les protecteurs de la nation italienne. Ainsi, plutôt que de louer les hautsfaits de Victor Emmanuel, Pascoli utilise cette description pour faire du roi un monument symbolisant le Risorgimento et protégeant l'Italie pour les générations à venir. 


\section{Victor Emmanuel, garant d'un nouvel âge d'or}

Nous avons vu que Pascoli utilisait le procédé de la superposition dans la description de la statue, associant Victor Emmanuel aux héros fondateurs de l'histoire de l'Italie. Mais le rapprochement le plus évident est celui effectué avec Auguste. En effet, le roi est représenté comme le garant de la paix par la guerre, comme le confirme la deuxième strophe qui décrit le retour au calme: Renidet / Pax roseo ueneranda caelo, le ciel rose symbolisant le sang versé27. En outre, en désignant l'Autel de la Patrie du nom d'Ara pacis (v. 9), Pascoli le superpose au monument inauguré par Auguste en 9 av. J.-C. ${ }^{28}$. Cette association résulte aussi de la publicité du gouvernement, qui donna au roi le titre de Padre della Patria - comme le Sénat l'avait fait pour Auguste - et qui fit graver ce titre sur sa tombe au Panthéon.

21 Commence alors la prière du poète pour l'avènement d'un nouvel âge d'or développée dans les strophes 3 à 6 , dans laquelle nous retrouvons les souhaits et les éléments d'utopie, un mode d'expression caractéristique des miroirs du Prince ${ }^{29}$. Pascoli reprend les codes de la poésie augustéenne, notamment l'emploi de la liste des bienfaits de l'âge d'or que l'on trouve dans Verg., Ecl. 4, 18 sq., ou Hor., Epod. 14, 42 sq. Il s'agit d'une liste de souhaits au subjonctif dont la première partie (strophe 3), à propos de la fertilité de la terre, rappelle les prières d'Horace dans Carm. saec. 29-32:

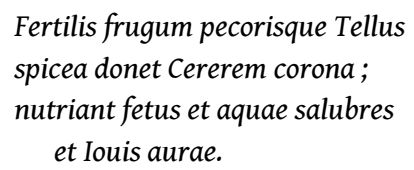

«Que la Terre, mère fertile des moissons et du bétail, décore Cérès d'une couronne d'épis; que les eaux et les souffles salubres de Jupiter nourrissent ce qu'elle enfante » [trad. F. Villeneuve].

Cependant, à la production spontanée de ressources qu'offre la terre chez les poètes augustéens ${ }^{30}$, Pascoli oppose le modèle de la terre qui récompense le travail, avec la personnification du latius raster ${ }^{31}$ : cet âge d'or moderne repose sur le travail, et s'inscrit plus explicitement dans les strophes suivantes dans un contexte de révolution industrielle et de luttes sociales, tout en s'inspirant du vocabulaire et des modèles antiques.

Ainsi, puisque Victor Emmanuel II semble être un symbole plus qu'un monarque à qui transmettre ses souhaits ou conseils, c'est plutôt à l'ensemble des Italiens que Pascoli tend ce miroir, célébrant les réalisations du roi mais aussi de l'Italie et rêvant à la prospérité de celle-ci. Ces vœux deviennent alors une évocation des grandes problématiques idéologiques qui traversent la politique italienne de son époque et sur lesquelles Pascoli prend position.

\section{Célébrer pour définir : un miroir de la nation italienne}

\section{Prières de Pascoli pour un âge d'or de l'Italie}

Le retour de l'âge d'or semble, pour Pascoli, reposer sur le travail, en ce qu'il permet l'accès à la petite propriété. Tout en évoquant les machines à vapeur et les pistons (altis sibilis et fumis) caractéristiques de la révolution industrielle, il attribue au travail une épithète épique, ignipotens, «maitre du feu », qui désigne Vulcain (Verg., A. 8, 414 sq.).

Interférences, 11 | 2018 
La petite propriété rurale est également inspirée d'un modèle antique : la maison sabine offerte par Mécène à Horace, qui l'a méritée par son travail de poète ${ }^{32}$.

La question du travail est intimement liée à la problématique de l'émigration italienne. L'Italie, victime du chômage, compte de très grandes disparités sociales. Dans le milieu agricole, la spéculation sur les grands domaines fait monter les prix et empêche l'accès à la petite propriété ${ }^{33}$. La strophe 5 est alors l'occasion de souhaiter une amélioration des conditions de vie des émigrés italiens exsules, soliuagos et inopes iuris, "exilés ", «solitaires » et «privés de droits». De ces conditions de vie déplorables Pascoli fera quelques mois plus tard son premier argument en faveur de l'expédition coloniale en Libye dans La grande proletaria si è mossa $a^{34}$, considérant que l'obtention d'une parcelle en Libye permettrait aux Italiens de ne pas être apatrides. Ce poème contient donc les prémices de ce discours, qui représentera d'ailleurs aussi l'Italie comme une «santa madre nostra Italia ", « madre d'ogni umanità » (" notre sainte mère l'Italie », " mère de toute humanité »), affligée par l'exploitation de ses fils, et s'affirmant en conquérante et en garante des anciennes valeurs romaines à travers le monde. Cet âge d'or relance ainsi la dynamique des conquêtes pour que l'Italie devienne une nation compétitive sur le plan géopolitique, mais aussi dans les domaines du commerce et des avancées techniques (avec la périphrase ueliuolum [...] caelum pour désigner l'avion, nouveau moyen d'exploration).

Les strophes 7 et 8 concernent quant à elles l'éducation, qui repose sur les valeurs anciennes (l'obéissance, la pudeur, la mesure et les mœurs anciennes) que l'on retrouve dans Hor., Carm. saec.57-60. Ces vertus correspondent d'ailleurs à celles que l'on loue chez le dirigeant dans l'Antiquité, ce qui en fait des topoi de l'éloge ${ }^{35}$. La démarche de Pascoli semble donc exalter l'identité italienne héritière de ces valeurs. En outre, l'éducation relève de l'instruction publique, la grande réalisation sociale de l'État unifié avec la loi Casati. L'emploi du terme Quirites n'est pas anodin, puisqu'il donne aux jeunes Italiens le nom des citoyens romains, associé aux récits fondateurs de Rome et à Romulus-Quirinus. L'école publique semble donc participer à la construction de l'identité nationale qui repose sur les valeurs romaines et - c'est en tout cas le souhait de Pascoli - sur l'enseignement du latin, bien que celui-ci ne soit pas accessible à tous ${ }^{36}$. L'enseignement du latin et de la langue italienne dans les établissements publics assure une transmission du patrimoine culturel italien, obéissant ainsi à la devise «Fatta l'Italia, ora bisogna fare gli Italiani » que Pascoli reprend notamment pour conclure La grande proletaria si è mossa.

27 Ainsi, c'est en s'inspirant de mots et de modèles anciens que Pascoli rêve de l'Italie idéale, héritière des valeurs romaines mais engagée dans des problématiques sociales modernes. Ces prières sont en effet l'occasion de dresser le bilan des réalisations de l'Italie au cinquantenaire de son unification, d'aborder les grandes questions idéologiques de son époque et de prendre position à leur sujet, une position socialiste et patriote. La forme adoptée pour porter un tel discours idéologique, rapprochant l'Italie de ses origines latines, sert quant à elle à rappeler sa légitimité.

\section{L'identité retrouvée par la guerre}

En effet, l'usage du latin et l'emploi de termes empruntés aux poètes augustéens ne servent pas seulement le but esthétique qui consiste à reprendre les codes des miroirs du Prince de l'Antiquité pour transmettre à l'État ou au public des revendications 
contemporaines. Ils sont un moyen de renouer avec les racines de la nation italienne, désormais pleinement assumées puisque Rome fait partie du royaume d'Italie. Pascoli rappelle cette continuité dans l'ensemble du poème, avec l'emploi des mots latius raster, « râteau latin » (strophe 3), Quirites pour désigner les citoyens (strophe 8) ou Latini pour désigner le peuple, ou encore antiqua mater pour désigner la patrie.

L'« Italie » renvoie alors à deux entités : la patrie, terre des ancêtres, ici représentée par une figure maternelle qui protège ses citoyens et les nourrit (altrix Italia), et la nation, fondée sur un sentiment d'appartenance qui dépasse le simple fait d'habiter en Italie puisqu'elle concerne aussi les «exilés ( (exsules) et les marins (nostri nauitae). Or, c'est dans la guerre que l'Italie a pu vraiment connecter ces deux entités et ainsi assumer pleinement son identité. Pascoli la représente d'ailleurs comme prête à combattre à nouveau, invoquant comme ses ancêtres le dieu Mars en frappant son bouclier et ses armes (comme dans Verg., A. 8,3 et 12,132). C'est pourquoi on peut supposer que ce que contemplent d'en haut les deux défenseurs (geminus uindex), c'est la nation italienne, qui a retrouvé ses droits sur sa patrie, qui s'est reconnectée à son passé, et qui est désormais prête soit à se défendre, soit à entreprendre des guerres coloniales, puisque Pascoli considère que la Libye serait une extension du territoire de la patrie.

D'après Jean-Philippe Genet, « les miroirs ne sont pas seulement tendus au prince : ils le sont à l'ensemble de la société politique ${ }^{37}$ ». Or, au début du $\mathrm{xx}^{\mathrm{e}}$ siècle, le Royaume d'Italie est une monarchie constitutionnelle, ce qui entraîne un élargissement de la société politique et change le rapport du roi à sa cour et à ses conseillers. Pourquoi alors écrire un miroir du Prince en 1911 ?

31 À l'occasion de l'inauguration de la statue équestre de Victor Emmanuel II et de l'Autel de la Patrie, Pascoli a proposé un poème-monument. En décrivant la statue du roi, et en espérant la venue d'un nouvel âge d'or, il célèbre les actions du roi et de ses troupes lors de l'unification italienne et les hauts-faits à venir, qui incombent à ses successeurs. Il s'agit donc d'un miroir indirect puisque le grand-père sert a priori d'exemple au petitfils, dans une logique de continuité dynastique. Cependant, cette description d'un monument posthume fait de Victor Emmanuel II et de Garibaldi des figures tutélaires et protectrices de la nation, les premiers exemples italiens d'un peuple récemment uni, c'est-à-dire un miroir du peuple. Pascoli disposant de moyens d'expression assez libres puisqu'il ne fait pas partie des cercles de la cour, nous avons supposé qu'il utilisait les codes du miroir du Prince pour véhiculer ses idéaux concernant la société italienne. De fait, l'ensemble des souhaits qu'il formule pour l'Italie sont l'occasion de dresser un bilan des réalisations de l'Unité italienne et de faire renouer la nation italienne avec ses origines latines. L'Italie s'étant révélée à elle-même par les guerres d'unification, il s'agit également de représenter une nation prête à se battre pour affirmer son identité dans les défis géopolitiques. Ce poème, qui aborde les grandes questions idéologiques qui traversent la politique italienne de l'époque de Pascoli, est aussi pour l'auteur un moyen de prendre position par rapport à celles-ci, et d'assumer un rôle de poeta vate socialiste et patriote. Le fait de transformer le modèle ancien de l'ode de célébration dans ce but atteste des transformations du rôle de la poésie politique à l'heure du développement de réflexions sur le concept de nation : elle ne négocie plus une adresse 
au monarque mais exalte le lien entre les décisions du gouvernement et ses conséquences sur les Italiens.

\section{BIBLIOGRAPHIE}

\section{Textes anciens}

Horace, Odes et épodes, texte établi et trad. par F. VILLENEUVE, 6 éd., CUF, Paris, Les Belles lettres, 1959.

- Satires, texte établi et trad. par F. VILLENEUVE, CUF, Paris, Les Belles lettres, 1962.

Virgile, Bucoliques, texte établi et trad. par E. DE SAINT-DENIS, $7^{\mathrm{e}}$ éd. du cinquième tirage rev., corr. et augm. d'un complément bibliographique, CUF, Paris, Les Belles lettres, 2003.

- Énéide. I, Livres I-VI, texte établi par H. GOELZER et trad. par André BELLESSORT, CUF, Paris, Les Belles lettres, 1942.

- Énéide. II, Livres VII-XII, texte établi par R. DURAND et trad. par A. BELLESSORT, CUF, Paris, Les Belles Lettres, 1957.

- Géorgiques, texte établi et trad. par E. DE SAINT-DENIS, CUF, Paris, Les Belles Lettres, 2003.

\section{Textes de G. Pascoli et P. Villari}

PASCOLI G. 1895, Lyra romana, ad uso delle scuole classiche, Livourne, R. Giusti.

- 1897, Epos, Livourne, R. Giusti.

- 1906, Odi e inni, Bologne, N. Zanichelli.

- 1908, Le canzoni di re Enzio, Bologne, N. Zanichelli.

- 1911a, Ad victorem regem: anno Italiae liberatae L, Bologne, N. Zanichelli.

- 1911b, Hymnus in Taurinos: anno ab Italia in libertatem vindicata quinquagesimo, testo latino e trad. italiana, Bologne, N. Zanichelli.

- 1911c, Hymnus in Romam anno ab Italia in libertatem vindicata quinquagesimo, testo latino e trad. italiana, Bologne, N. Zanichelli.

- 1911d, La grande proletaria si è mossa : discorso tenuto a Barga « Per i nostri morti e feriti », Bologne, N. Zanichelli.

- 1911e, Poemi italici, Bologne, N. Zanichelli.

- 1913, Poemi del Risorgimento: Inno a Roma, Inno a Torino, Bologne, N. Zanichelli.

- 1914, « Un poeta di lingua morta », in Pensieri e discorsi, Bologne, N. Zanichelli. URL : http:// www.classicitaliani.it/pascoli/prosa/pascoli_poeta_lingua_morta.htm. 
- 1995, Post Occasum Urbis, a cura di O. AIELLO, Biblioteca philologica 2, Palerme, L'Epos.

- 2011, Pascoli, Tutte le poesie, a cura di A. COLASANTI, trad. e note delle Poesie latine die N. CAlzolaio, I Mammut 72, Rome, Newton Compton.

VILLARI P. 1909, Scritti sulla emigrazione e sopra argomenti vari, Bologne, N. Zanichelli.

\section{Études modernes}

BODART D. 2012, « Le Prince miroir, métaphore optique du corps politique », in P. Morel (dir.), Le miroir et l'espace du prince dans l'art italien de la Renaissance, Renaissance, Tours, Presses universitaires François-Rabelais de Tours ; Rennes, Presses universitaires de Rennes, p. 123-142. CORBI E., SARRACINO V. 2003, Scuola e politiche educative in Italia dall'Unità a oggi, Domini 1, Naples, Liguori.

HADOT P. 1972, «Fürstenspiegel », in Das Reallexikon für Antike und Christentum, VIII, Stuttgart, A. Hiersemann, p. 555-632.

LACHAUD F, SCORDIA L. (dir.) 2007, Le Prince au miroir de la littérature politique de l'Antiquité aux Lumières, Mont-Saint-Aignan, Publications des universités de Rouen et du Havre.

PASCOLI M. 1961, Lungo la vita di Giovanni Pascoli, Memorie curate e integrate da A. VICINELLI, con 48 tavole fuori testo, Milan, A. Mondadori. URL : http://www.classicitaliani.it/index228.htm.

PERNOT L. 1993, La rhétorique de l'éloge dans le monde gréco-romain. I, Histoire et technique, Coll. des études augustiniennes. Série Antiquité 137, Paris, Institut des études augustiniennes.

TRAINA A. 2006, Il latino del Pascoli : saggio sul bilinguismo poetico, Testi e manuali per l'insegnamento universitario del latino 88, Bologne, Pàtron.

Giovanni Pascoli nello specchio delle sue carte. L'archivio e la casa di Giovanni e Maria Pascoli a Castelvecchio. URL : http://pascoli.archivi.beniculturali.it/index.php?id=9.

\section{NOTES}

\section{PASCOLI 2011, p. 1202.}

2. Deux hendécasyllabes, un ennéasyllabe et un décasyllabe alcaïque. Pascoli a composé de nombreuses odes de circonstance, dédicacées à des personnalités politiques ou culturelles et inspirées d'Horace, aussi bien du point de vue de la variété métrique que du contenu.

3. La coupure du journal est accessible sur le site de l'Archivio di Castelvecchio, cote P. 9.1.26.

4. PASColi 1911a. L'édition illustrée est également en ligne sur le site de l'Archivio di Castelvecchio, cote G.83.3.3.

5. Cette section fait à présent partie des œuvres complètes : PASCOLI 2011.

6. Cf. M. PASCOLI 1961, titre du chap. II de la IV e partie : « Il 1911: l'anno sacro » <http:// www.classicitaliani.it/pascoli/pascolim_13.htm>.

7. PASCOLI 1913. Pascoli étant tombé malade à la fin de l'année 1911, la publication fut reportée. Elle intégra les deux hymnes latins et leur traduction par le poète. 
8. PASCOLI 1911 b; PASCOLI 1911c.

9. PASCOLI 1911d.

10. Cf. M. PAscoli 1961, titre de la IV e partie de l'ouvrage : « La turbata vita bolognese e il momento epico-storico della poesia (1906-1911)».

11. PASCOLI 1906 ; PASCOLI 1908 ; PASCOLI 1911e.

12. PASCOLI 1995, p. XIX.

13. M. PASCOLI 1961, partie IV, chap. I < http://www.classicitaliani.it/pascoli/ pascolim_12.htm>.

14. Cette épigraphe est reproduite par des coupures de journaux disponibles sur le site de l'Archivio di Castelvecchio, cote G.77.2.11.

15. Sur le développement sur la situation d'énonciation caractéristique des miroirs du Prince, voir l'Introduction de ce numéro d'Interférences.

16. PASCOLI 1914.

17. PASCOLI 1895 ; PASCOLI 1897.

18. PASCOLI 2011, respectivement p. 1108-1123, p. 1124-1135 et p. 914-924.

19. PASCOLI 2011, p. 924-934

20. TRAINA 2006, p. 226.

21. Sur le développement sur l'éloge, voir l'Introduction de ce numéro d'Interférences.

22. HADOT 1972 opère ce rapprochement avec la littérature panégyrique, notamment le Panégyrique de Trajan pour la littérature latine (p. 609). L'introduction de l'ensemble des articles étudie la pertinence de ce rapprochement en poésie latine.

23. Voir BODART 2012, p. 126.

24. En 1911 il s'agissait de l'original, que l'on retrouve à présent à l'intérieur des musées Capitolins.

25. On retrouve ce motif dans le poème «I due fanciulli » (Primi poemetti, in PASCOLI 2011, p. 150) où deux jumeaux qui se battent sont séparés par leur mère puis s'endorment réconciliés. Cette superposition des fratries n'est pas anodine puisque la relation entre Garibaldi et Victor Emmanuel a été ponctuée de désaccords (au sujet de l'annexion de Nice à la France ou de celle de Rome au Royaume d'Italie par ex.).

26. Pascoli a certainement voulu rapprocher les deux fratries parce que Romulus et Rémus sont associés à la constellation des Gémeaux à Rome comme Castor et Pollux le sont en Grèce.

27. Pascoli emploie la même image après la rixe entre Romulus et Rémus dans l'Hymnus in Romam, v. 110 : in roseo dubius tremis, Hespere, caelo, « hésitant tu trembles dans le ciel rose, Hespérus ». D'après Alfonso Traina, il s'agit d'une image typiquement pascolienne de ces moments de trépas, quand coexistent et se fondent l'un dans l'autre ce qui va mourir et ce qui vient de naître (TRAINA 2006, p. 63).

28. Si le musée de l'Ara Pacis n'existait pas encore, le monument avait été découvert au $\mathrm{XVI}^{\mathrm{e}} \mathrm{s}$. et identifié à la fin du XIX pour ensuite être reconstitué les années suivantes. Il était cependant connu grâce aux sources (notamment Ov., Fast. 1, 709 sq., et Aug., Anc. 2, 37).

29. HАDOT 1972, p.566. Cet article propose une réflexion sur les origines antiques du miroir du Prince et repère des codes littéraires et des topoi communs aux miroirs du 
Prince médiévaux et à des textes antiques adressés au dirigeant bien qu'ils ne portent pas encore le nom de miroir. Parmi ces codes, P. Hadot signale l'utilisation de l'éloge et de procédés didactiques, la formulation de souhaits, la présence d'éléments de biographie et de situations utopiques. Ces notions sont développées à propos de la poésie dans l'Introduction de ce numéro d'Interférences.

30. Inarata, « sans labour ", écrit Horace (Hor., Epod. 16, 43), et nullo cultu, « sans aucune culture ", Virgile (Verg., Ecl. 4, 18), qui se plait ensuite à utiliser le pronom ipse dans l'ensemble de la description.

31. Pascoli semble préférer comme modèle l'épisode du vieillard de Tarente (Verg., G. 4, 125-146) qu'il réécrit dans son poème Senex Corycius (1902, in PASCOLI 2011, p. 962-970), en mettant en scène sa rencontre avec Virgile (Senex Corycius, v. 48-52) : [...] niveaque senex per gramina barba / reptat, et hic fulcit pendentia lilia iunco, / hic paleis operit rugosam frigore caltham, / hic cratem tendit duplici super amite virgeam, / ut possit gelidas contemnere flosculus auras, " un vieillard à la barbe d'un blanc de neige, à travers les plantes / marche avec lenteur, et ici soutient des lis qui pendaient avec un jonc, / ici de paille il recouvre, ridée par le froid, un souci, / ici il étend sur deux bâtons une claie faite de brindilles, / pour qu'elle puisse, la jeune fleur, faire fi des brises glacées» [trad. personnelle].

32. Fanum Vacunae (1910) raconte par ex. l'arrivée d'Horace dans la villa (PASCOLI 2011, p. 940-962) ; Moretum (1900) met en scène Mécène qui discute avec Horace de la morale rurale et de la petite propriété, en s'inspirant de Hor., S. 2, 6, $1:$ Hoc erat in uotis : modus agri non ita magnus, / hortus ubi et tecto uicinus iugis aquae fons / et paulum siluae super his foret, «C'était mon vœu: un domaine dont l'étendue ne serait pas trop grande, où il $\mathrm{y}$ aurait un jardin, une fontaine d'eau vive voisine de la maison, et, au-dessus, un peu de bois» (trad. F. Villeneuve).

33. Pascoli est très engagé sur le sujet de l'émigration italienne. Le poème latin Ecloga XI (1908) est à rapprocher du poème italien "Pietole» (1909, Nuovi poemetti, in PASCoLI 2011, p. 257-264), dans lequel l'esclave fugitif d'Ecloga XI est comparé à l'Italien qui décide d'émigrer et qui répète dans diverses langues «je suis italien, j'ai faim». Ce poème est d'ailleurs accompagné de citations de P. VILLARI (1909) dont Pascoli soutient les projets de ventes de parcelles à prix juste.

34. «Il mondo li aveva presi a opra, i lavoratori d'Italia; e più ne aveva bisogno, meno mostrava di averne, e li pagava poco e li trattava male [...] ", « Le monde les avait pris comme main d'œuvre, les travailleurs d'Italie; et plus il en avait besoin, moins il montrait que c'était vrai, et il les payait peu et les traitait mal » (PASCOLI 1911d).

35. Ces topoi sont étudiés en détail dans PERNOT 1993, p. 134-178. Dans ce chapitre, Laurent Pernot synthétise et illustre les travaux des maîtres de la rhétorique sur la composition d'un discours d'éloge, et notamment ceux de Ménandre le Rhéteur qui a établi des listes de topoi, parmi lesquels figurent les vertus. Celles-ci sont également l'objet de listes inspirées de l'amalgame entre l'éthique platonicienne, les bonnes mœurs, et les valeurs que veut promouvoir le régime en place. Voir le développement que nous en faisons dans l'Introduction de ce numéro d'Interférences.

36. Le latin reste en effet enseigné seulement dans les formations d'élite (« ginnasi » et lycées classiques). D'autre part, seule l'école élémentaire était gratuite (CORBI, SARRACINO 2003, p. 24).

37. LACHAUD, SCORDIA (dir.) 2007, p. 423. 


\section{RÉSUMÉS}

Ad Victorem regem, poème composé par Giovanni Pascoli en 1911, ressemble à un miroir du Prince. S'inspirant des poètes augustéens, il célèbre les hauts-faits de Victor Emmanuel II lors du Risorgimento et lui adresse des vœux pour la prospérité de l'Italie. Cependant, le premier roi de l'Italie est mort depuis 23 ans lorsque Pascoli écrit ce texte et, dans le cadre d'une monarchie constitutionnelle, le miroir du Prince ne semble plus être le mode d'adresse au dirigeant le plus adapté. Pourquoi alors recourir à ce genre ? Nous verrons que ce texte semble plutôt être adressé aux Italiens et qu'il reprend les codes du miroir du Prince pour célébrer l'unité italienne, faire le bilan de ses cinquante ans d'existence, légitimer la nation italienne par ses origines latines et espérer son plein épanouissement.

Ad Victorem regem, poem written by Giovanni Pascoli in 1911, looks like a mirror for Princes. Inspired by the Augustan poets, it celebrates the accomplishments of Victor Emmanuel II during the Risorgimento and makes wishes for the prosperity of Italy. But the first king of Italy has been dead for 23 years when Pascoli writes this text, and the mirror for Princes doesn't seem to be the most adapted way to speak to a ruler in a context of constitutional monarchy. Why then did Pascoli write a mirror for Princes? We'll see that this text is rather addressed to the Italians and that it uses the mirror's codes to celebrate the Italian Unity, assesses its accomplishments after 50 years, legitimates the Italian nation with its Latin origins and hopes for it's prosperity.

\section{INDEX}

nomsmotscles Claudien, Horace, Marc Aurèle, Virgile

Keywords : golden age, celebration, poetry, Risorgimento, Victor-Emmanuel II, VictorEmmanuel III, power, mirror of Princes

Index géographique : Italie, Libye, Lucques, Rome

Mots-clés : âge d'or, célébration, poésie, pouvoir, Risorgimento, Victor-Emmanuel II, Victor-

Emmanuel III, miroir du Prince

\section{AUTEURS}

\section{SARAH ORSINI}

Université Lumière Lyon 2 - Università degli Studi di Roma Tre, UMR 5189 HiSoMA 